\title{
Inter-visit measurement variability of conjunctival vasculature and circulation in habitual contact lens wearers and non-lens wearers
}

\author{
Jianhua Wang ${ }^{1}$, Liang Hu ${ }^{1,2}$, Ce Shi $^{1,2}$ and Hong Jiang ${ }^{1 *}$ (D)
}

\begin{abstract}
Background: The inter-visit variation of measuring bulbar conjunctival microvasculature and microcirculation needs to be considered when the results from multiple visits are interpreted. This study examined the inter-visit variability of measuring conjunctival microvasculature and microcirculation in habitual contact lens (HCL) wearers and non-contact lens (NCL) wearers.

Methods: Twenty-eight subjects were recruited including $13 \mathrm{HCL}$ wearers (10 females and 3 males; mean age \pm standard deviation, $25.8 \pm 4.6$ years) who had worn contact lenses on a daily basis for at least 3 years and $15 \mathrm{NCL}$ wearers (10 females and 5 males, age $25.5 \pm 4.0$ years) were recruited. The temporal bulbar conjunctiva was imaged using a functional slit-lamp bio-microscope (FSLB) imaging system. FSLB imaging was performed in the morning when the $\mathrm{HCL}$ wearers did not wear their lenses. The measurements included conjunctival vessel diameter, vessel density, blood flow velocity and flow volume. In addition, conjunctival microvasculature was analyzed using monofractal (Dbox, representing vessel density) and multifractal (D0 representing vessel complexity) analyses. The repeated measurement was conducted at least one week after the first visit and both eyes of each participant were imaged. The coefficient of variation (CV) was calculated as the standard deviation of the differences between test and re-test then divided by the mean of the measurements. The intra-class correlation coefficient (ICC) was also calculated.
\end{abstract}

Results: No significant differences of all vascular measurements in both the right and left eyes were found between two groups $(P>0.05)$. Between two measurements on two different visits, the $\mathrm{CV}$ was from $2.4 \%$ (vessel density D0) to 63.5\% (blood flow volume Q) in $\mathrm{HCL}$ wearers and from 3.4\% (D0) to $40.6 \%$ (blood flow volume) in NCL wearers. The ICC was from 0.60 (vessel diameter) to 0.81 (axial blood flow velocity VA) in $\mathrm{HCL}$ wearers and from 0.44 (Q) to 0.68 (cross-sectional blood flow velocity VS) in NCL wearers.

Conclusions: The measurement variability of the vessel density of the bulbar conjunctiva appeared to have the smallest inter-visit variation. The measurement variability of the vasculature and circulation in $\mathrm{HCL}$ wearers were similar to that in NCL wearers.

Keywords: Variability, Bulbar conjunctiva, Blood flow velocity, Microvascular network, Functional slit-lamp biomicroscopy (FSLB), Hemodynamics

\footnotetext{
* Correspondence: h.jiang@med.miami.edu

${ }^{1}$ Miller School of Medicine, Bascom Palmer Eye Institute, University of Miami,

1638 NW 10th Avenue, McKnight Building - Room 202A, Miami, FL 33136,

USA

Full list of author information is available at the end of the article
}

(c) The Author(s). 2019 Open Access This article is distributed under the terms of the Creative Commons Attribution 4.0 International License (http://creativecommons.org/licenses/by/4.0/), which permits unrestricted use, distribution, and reproduction in any medium, provided you give appropriate credit to the original author(s) and the source, provide a link to the Creative Commons license, and indicate if changes were made. The Creative Commons Public Domain Dedication waiver (http://creativecommons.org/publicdomain/zero/1.0/) applies to the data made available in this article, unless otherwise stated. 


\section{Background}

When a contact lens is placed on the ocular surface, alterations to the vasculature take place immediately in response. Contact lens wear induces ocular surface indentation, especially on the conjunctiva, which causes mechanical pressure to the ocular surface. Wearing a contact lens can disturb the integrity of the tear film and forms two distinct tear films, one between the lens and eye, and another above the lens, which further induces possible friction on the ocular surface. In addition, contact lens wear induces some level of low oxygen supply, although lenses with high oxygen transmission materials (i.e., silicone hydrogel) [1-3] may already eliminate the issue with daily wear of contact lenses. When the eyelid is open, oxygen from the ambient air diffuses into the bulbar conjunctival microvasculature, which highly oxygenates all exposed micro-vessels within $10 \mathrm{~s}$ [4]. However, the situation may change if the contact lens covers some portion of the conjunctiva, resulting in vascular responses. In neophytes who have never had experience with contact lenses, contact lens wear induced a 30\% increase of bulbar conjunctival blood flow velocity after $6 \mathrm{~h}$ of lens wear [5]. Bulbar conjunctival blood flow velocity in habitual lens wearers was about $22 \%$ higher than normal controls when they wore their lenses [6]. The alteration of the vessel density (Dbox) in the bulbar conjunctiva in habitual lens wearers was found to be about $3 \%$, which was significant compared to controls. As noted from our previous study, these habitual lens wearers did not notice that their bulbar conjunctivas appeared to be slightly red. These subtle changes may indicate an ongoing response to contact lens wear. Therefore, a precise evaluation of the vascular responses to contact lens wear is critical for studying modern contact lenses. There is a knowledge gap in the inter-visit measurement variation, which is essential for calculating sample sizes and study designs of clinical research on contact lenses.

With the introduction of new contact lens materials (e.g., silicone hydrogel) and lens designs, contact lens-related complications have been dramatically reduced [7-11]. However, some contact lens-associated changes on the ocular surface such as conjunctival hyperemia, corneal infiltration and microbial keratitis often occur $[8,9,12-15]$. While the attention has been shifted to ocular comfort [16], vascular responses to contact lens wear is still a hot topic which can provide better understanding of the underlying mechanism of ocular discomfort associated with contact lens wear $[5,6,11,17-20]$. Previous evaluation using slit-lamp photography for evaluating the redness of the bulbar conjunctiva was found to have poor measurement variation [2, 3, 21]. Indeed, bulbar conjunctival hyperemia is an obvious sign of vascular response to contact lens insertion [2, 11, 18, 22, 23]. In addition to bulbar conjunctiva redness, blood flow in the vessels alters in concert with the vessel network change, which provides an opportunity for studying the circulation response to contact lens wear $[5,6,19,20]$. The alterations of conjunctival blood flow velocity in contact lens wearers have been well documented in previous studies [5, 6, 19]. Precise measurements of conjunctival microvasculature (i.e., vessel diameter and density) and microcirculation (i.e., blood flow velocity and flow rate) are crucial in evaluating physiological responses to contact lenses, which often require measurements to be taken during multiple visits on different days [20]. Therefore, the inter-visit variation of these measurements needs to be considered when the results from multiple visits are interpreted. The goal of this study was to examine the inter-visit variability of measuring conjunctival microvasculature and microcirculation in habitual contact lens (HCL) wearers and non-contact lens (NCL) wearers.

\section{Methods}

This study was approved by the Human Subject Research Office (HSRO) at the University of Miami (ID: 20150359) and every participant signed an informed consent form. The study was conducted in accordance with the tenets of the Declaration of Helsinki. Similar to the system used in previous studies by others [17, 24-26], a functional slit-lamp biomicroscope (FSLB) was developed and fully validated [27]. FSLB has been used in previous studies and the procedures are well described [5, 19, 27, 28]. The system applies a high-speed camera which has a video cropping function that enables the addition of a $7 \times$ digital magnification into the slit-lamp. This allows a total magnification of up to $210 \times$, which is sufficient for imaging a cluster of red blood cells. With high-speed video capturing, blood flow can be recorded and analyzed to obtain blood flow velocity and flow volume. Since blood flow velocity is different in arterioles $[25,29]$ and venules $[6,26,30]$, conjunctival venules were measured in the present study. Image processing procedures described in the previous studies were used to process the video clips $[5,19,26-28,30]$. The measurements included axial blood flow velocity (VA), cross-sectional blood flow velocity (VS) and flow volume (Q). The VA was measured by tracking the movement of red blood cells along the vessel [26]. The VS was calculated from VA, using a previously defined function that accounts for the diameter of the blood vessel (D) [30]. The cross-sectional flow rate, $\mathrm{Q}$, was determined using a standard flow rate equation: $\mathrm{Q}=\mathrm{VS} \pi \mathrm{D}^{2} / 4$ [30].

To measure vascular network density, a field of view of $0.9 \times 0.7 \mathrm{~mm}^{2}$ was used, and six fields of the temporal bulbar conjunctiva located $\sim 1 \mathrm{~mm}$ radially from the circumference of the limbus ring were imaged for 
measuring circulation (Fig. 1). In addition, vascular network in a field of $7.87 \times 7.87 \mathrm{~mm}^{2}$ was acquired (Fig. 2). Image processing procedures extracted the vessels which were skeletonized for fractal analysis using custom software which has been described previously [27]. The monofractal and multifractal analyses available in a software program (Benoit ${ }^{\mathrm{Tm}}$, TruSoft Inc., St. Petersburg, FL, USA) [27] were performed to evaluate the vessel density (Dbox,) and complexity (D0) of the conjunctival microvasculature. Fractal dimension has been used to estimate the branching pattern of the retinal vascular tree [31] and capillary network in the retina and conjunctiva $[27,32]$. Larger values indicate more dense vessels and more complex branching patterns.

Thirteen HCL wearers (10 females and 3 males; mean age \pm standard deviation, $25.8 \pm 4.6$ years) who had worn contact lenses on a daily basis for at least 3 years and 15 NCL wearers (10 females and 5 males, age $25.5 \pm 4.0$ years) were recruited. FSLB imaging was performed in the morning (from 8:30 am to $11 \mathrm{am}$ ) when the $\mathrm{HCL}$ wearers did not wear their lenses. The repeated measurement was conducted at least one week after the first visit and both eyes of each participant were imaged.

All data management and statistical analyses were carried out in Excel (v. 2010, Microsoft, Redmond, WA). The coefficient of variation $(\mathrm{CV})$ was calculated as the standard deviation of the differences between test and re-test then divided by the mean of the measurements. The Bland-Altman plot was used show the limits of agreement. The intra-class correlation coefficient (ICC) was also calculated using the Pearson correlation. The confidence interval of the $\mathrm{CV}$ was calculated as the $\mathrm{CV} \pm 1.96 \times \mathrm{CV} /$ square root $(2 \times$ sample size). The student's t-test was used to test the differences of vascular measurements between groups.
A $p$-value of less than $0.05(P<0.05)$ was considered statistically significant.

\section{Results}

No significant differences of all vascular measurements in both the right and left eyes were found between two groups $(P>0.05$, Table 1$)$. The Bland-Altman plots in the HCL (Fig. 3) and NCL (Fig. 4) groups showed the upper and lower limits of agreement (i.e., \pm 1.96 SD, 95\% confidence intervals). Between two measurements on two different visits, the $\mathrm{CV}$ ranged from $2.4 \%$ (vessel density D0) to $63.5 \%$ (blood flow volume, Q) in $\mathrm{HCL}$ wearers and from $3.4 \%$ (D0) to $40.6 \%$ (Q) in NCL wearers. The ICC was from 0.60 (vessel diameter) to 0.81 (VA) in HCL wearers and from $0.44(\mathrm{Q})$ to 0.68 (cross-sectional blood flow velocity VS) in NCL wearers.

\section{Discussion}

While the "true" values of these measured vascular parameters are unknown, the repeated measurements determine whether each of the measurements can be used interchangeably. In contact lens studies, repeated measurements are often needed when ocular responses to the lenses are being evaluated. Understanding the variation between repeated measurements will help interpret study results to determine whether the alteration is due to contact lens wear with different designs or materials. The CV of the blood flow velocity and vessel density found in this study is about the same as the changes due to contact lens wear documented in previous studies $[6,17]$, which provides a very good detection power (effect size $=\sim 1$ ) in determining whether the changes are true. This may explain the previous finding that significant changes were reached in a small cohort of contact lens wears compared to controls in neophytes

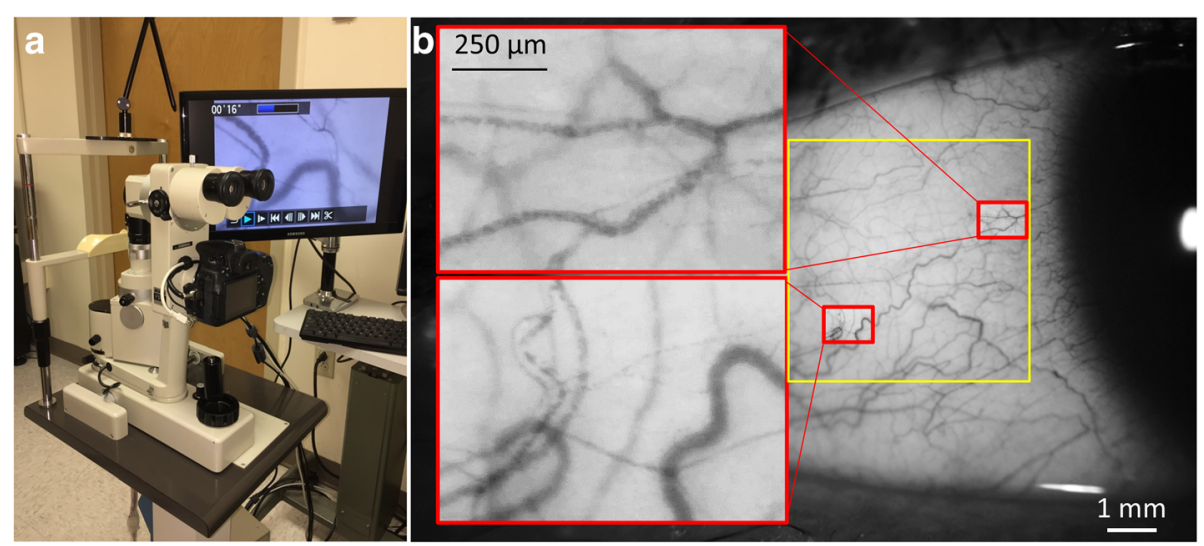

Fig. 1 Functional slit-lamp biomicroscope (FSLB) and images. FSLB was modified from a standard slit-lamp biomicroscope equipped with a digital camera through an inherent camera port (a). The video cropping function of the camera enables an additional 7× magnification, which is combined with the $30 \times$ inherent magnification. FSLB was used to acquire an image of the temporal conjunctiva (b) with high magnification, and the red blood cell clusters are shown in the inserts 

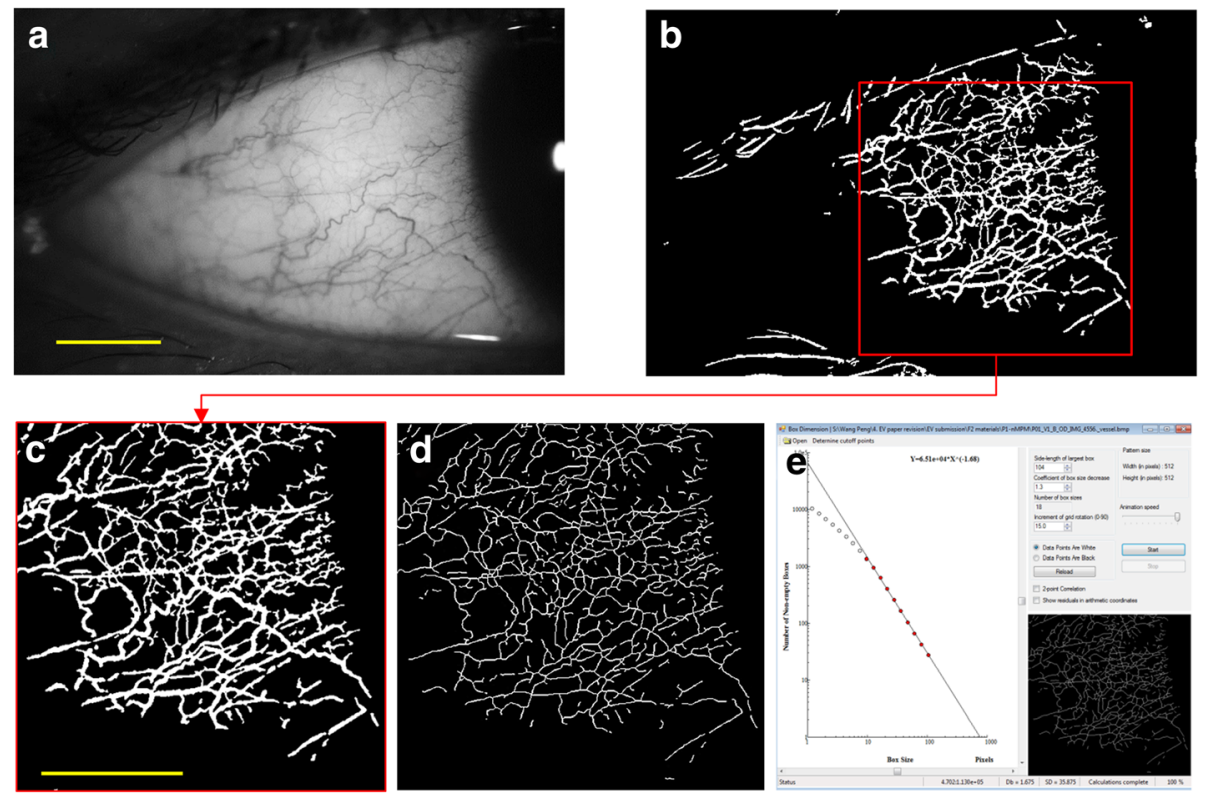

Fig. 2 Image processing of conjunctival vasculature and fractal analysis. Conjunctival vessels were segmented by using custom software to extract the conjunctival vessels for fractal analysis. The raw image was resized to $1024 \times 683$ pixels (a). Segmented vessels after using adaptive histogram equalization and morphological opening operations (b). Cropped image of $512 \times 512$ pixels with a field of view $7.87 \times 7.87 \mathrm{~mm}^{2}$ (c). Skeletonized vessel image (d). Fractal analysis of the skeletonized image (e). Bars $=3 \mathrm{~mm}$

Table 1 Vascular measurements of HCL $(N=13)$ and $\mathrm{NCL}(N=15)$ wearers in two visits

\begin{tabular}{|c|c|c|c|c|c|c|}
\hline & $\mathrm{D}(\mu \mathrm{m})$ & Dbox & DO & $\mathrm{Q}(\mathrm{pl} / \mathrm{s})$ & $\mathrm{VA}(\mathrm{mm} / \mathrm{s})$ & $\mathrm{VS}(\mathrm{mm} / \mathrm{s})$ \\
\hline \multicolumn{7}{|l|}{$\overline{\mathrm{HCL}}$} \\
\hline Visit 1 & $18.1 \pm 4.0$ & $1.64 \pm 0.04$ & $1.70 \pm 0.05$ & $150 \pm 119$ & $0.52 \pm 0.15$ & $0.37 \pm 0.10$ \\
\hline Visit 2 & $16.8 \pm 2.3$ & $1.63 \pm 0.06$ & $1.70 \pm 0.05$ & $118 \pm 62$ & $0.51 \pm 0.17$ & $0.37 \pm 0.12$ \\
\hline Diff & $1.3 \pm 3.2$ & $0.01 \pm 0.05$ & $0.00 \pm 0.04$ & $32 \pm 85$ & $0.01 \pm 0.10$ & $0.01 \pm 0.07$ \\
\hline Upper LOA & 7.65 & 0.10 & 0.08 & 199 & 0.21 & 0.15 \\
\hline Lower LOA & -5.03 & -0.08 & -0.08 & -135 & -0.18 & -0.14 \\
\hline ICC & 0.60 & 0.64 & 0.64 & 0.72 & 0.81 & 0.78 \\
\hline CV (\%) & 18.5 & 2.8 & 2.4 & 63.5 & 19.5 & 20.2 \\
\hline $\mathrm{Cl}$ of $\mathrm{CV}$ & $25.6 \sim 11.4$ & $3.9 \sim 1.7$ & $3.4 \sim 1.5$ & $88.0 \sim 39.1$ & $27.1 \sim 12.0$ & 27.9 12.4 \\
\hline \multicolumn{7}{|l|}{$\mathrm{NCL}$} \\
\hline Visit 1 & $17.2 \pm 2.4$ & $1.62 \pm 0.06$ & $1.69 \pm 0.06$ & $116 \pm 43$ & $0.49 \pm 0.13$ & $0.36 \pm 0.10$ \\
\hline Visit 2 & $16.8 \pm 2.6$ & $1.62 \pm 0.06$ & $1.70 \pm 0.06$ & $117 \pm 53$ & $0.51 \pm 0.16$ & $0.37 \pm 0.12$ \\
\hline Diff & $0.4 \pm 2.5$ & $0.00 \pm 0.06$ & $0.00 \pm 0.06$ & $-0.2 \pm 47$ & $-0.01 \pm 0.11$ & $-0.01 \pm 0.08$ \\
\hline Upper LOA & 5.3 & 0.12 & 0.11 & 93 & 0.20 & 0.14 \\
\hline Lower LOA & -4.5 & -0.13 & -0.12 & -93 & -0.22 & -0.16 \\
\hline ICC & 0.51 & 0.49 & 0.51 & 0.44 & 0.67 & 0.68 \\
\hline CV (\%) & 14.7 & 3.8 & 3.4 & 40.6 & 21.4 & 21.1 \\
\hline $\mathrm{Cl}$ of $\mathrm{CV}$ & $20.3 \sim 9.0$ & $5.3 \sim 2.4$ & $4.8 \sim 2.1$ & $56.3 \sim 25.0$ & $29.6 \sim 13.2$ & $29.2 \sim 13.0$ \\
\hline
\end{tabular}

$H C L=$ habitual contact lens wearers; $N C L=$ non-contact lens wearers; $D=$ diameter; $D b o x=$ fractal dimension processed using box counting indicating vessel density; $\mathrm{D} 0=$ multifractal dimension indicating vessel complexity; $Q=$ blood flow volume; $V A=$ axial blood flow velocity; $V S=$ cross-sectional blood flow velocity; $C V=$ coefficient of variation; $I C C=$ interclass correlation coefficient; $L O A=$ limit of agreement; $C l$ of $C V=$ confidence interval of $C V$ 

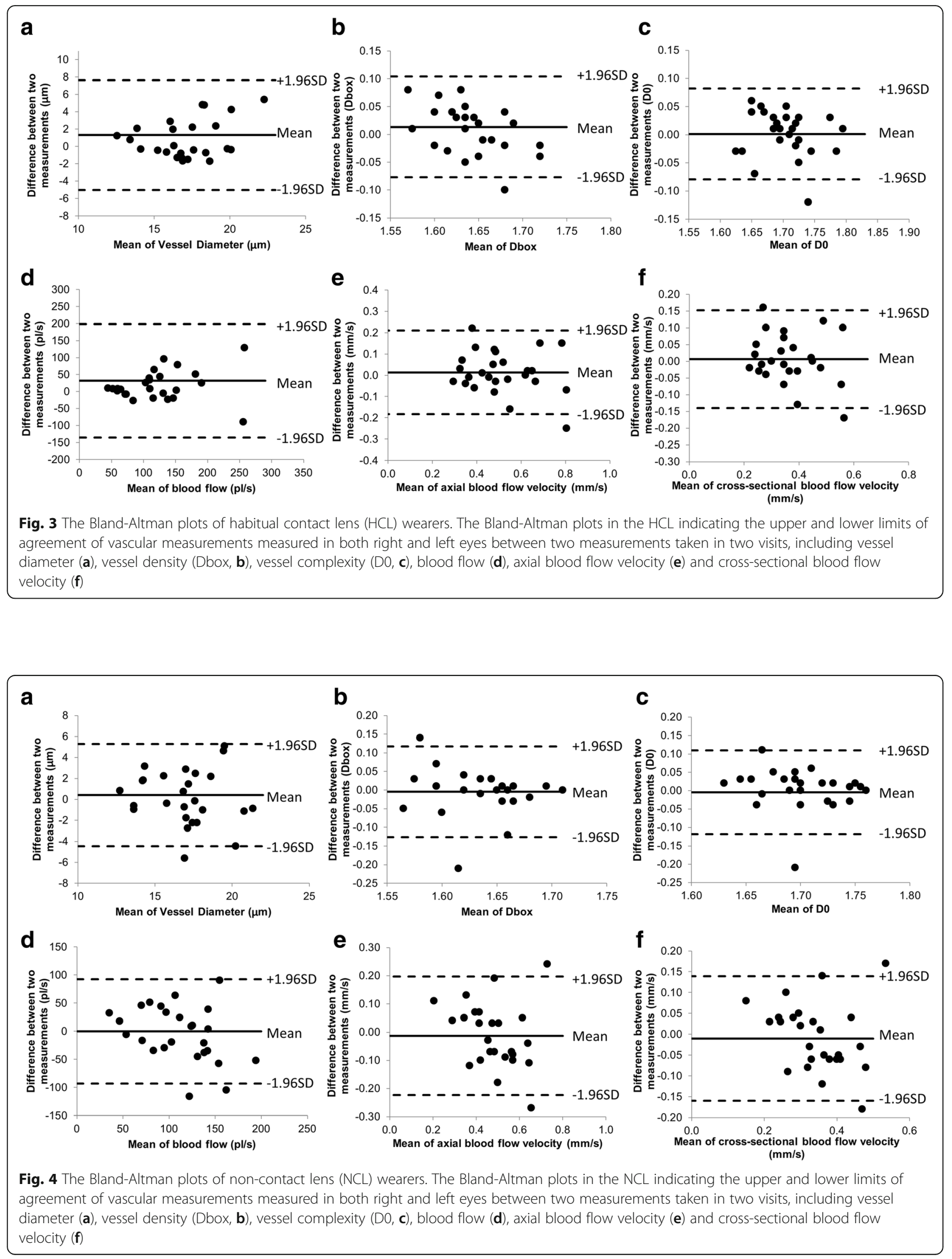
[5] and habitual lens wearers [6]. However, whether these parameters have sufficient power in differentiating the vascular responses to different lens designs and materials remain unknown. Thus, further studies are warranted.

The Bland-Altman plots showed all dots within the 95\% limits of agreement for vessel diameter and blood flow in the HCL, which may indicate good test-retest reliability in the $\mathrm{HCL}$. Interestingly, the $\mathrm{CV}$ and ICC were about the same between HCL and NCL groups, indicating that both groups had similar variation between visits and the methodology used in the present study yielded the expected results. The inter-visit variation appears to be similar to intra-visit variation demonstrated by $\mathrm{Xu}$ et al., who tested these vascular parameters at 9 $\mathrm{AM}$ and $11 \mathrm{AM}$ on the same day in a group of 20 healthy subjects, including habitual lens wearers and non-contact lens wearers [33]. In a recent inter-visit variability study conducted by Khansari et al., conjunctival hemodynamics of non-diabetic healthy subjects were measured repeatedly for 11 weeks, which was longer than the follow-up we did in the present study. Although a different image device was applied, the standard deviation of the difference between two visits was similar in conjunctival blood flow velocity $(0.16$ $\mathrm{mm} / \mathrm{s}$ vs. $0.11 \mathrm{~mm} / \mathrm{s}$ ).

However, the CV of measuring blood flow velocity of the conjunctiva is higher than the $\mathrm{CV}$ of measuring retinal blood flow velocity [34]. Burgansky-Eliash et al. reported that the intra-visit variability of measuring retinal blood flow was $11.2 \%$. The relatively large CV found in our study with two visits and the previous study with one visit for the measurements of blood flow velocity may be due to: 1) In fundamental ocular physiology, stable retinal blood flow is necessary to maintain the high metabolic activity of the retina for vision, whereas conjunctival blood flow is likely not as important for the conjunctiva. Further, retinal blood vessels are much more ordered than conjunctival blood vessels. Therefore, it is not surprising that conjunctival vessels have a larger $\mathrm{CV}$; 2) the ocular surface exposure to the outside environment such as temperature which may affect the circulation of the conjunctiva; 3) blinking which may exert mechanical pressure on the vessels; and 4) the small volumes of blood flow velocity (about $0.50 \mathrm{~mm} / \mathrm{s}$ ) which is about 6-8 times slower than that in the retina (about $3-4 \mathrm{~mm} / \mathrm{s}$ ), and may lead to the higher CV results.

There are some limitations to the present study. First, the measurement of blood flow velocity is not fully automatic and required the manual drawing of the slope in the time-space images as performed in previous studies. The inter-grader variation of the blood flow velocity measurements is reported to be about $5 \%$ which may have been included in inter-visit variability. Therefore, further development of fully automated image processing for measuring the velocity is needed. Second, while attention is given to relocate the same image locations on the conjunctiva, actual fields of view were not obtained because it was nearly impossible to get the same location during a video recording using extremely high magnification. Although we tried to image vessels with similar diameters, the measurements were not controlled by the vessel diameters in the present study. Vessel diameter correlates to blood flow velocity $[5,35]$. These factors in the selection of vessels and imaging location may contribute to the $\mathrm{CV}$, and further development on field tracking may be helpful. Third, only the venules on the conjunctiva were measured. Since blood flow velocity is different between the arterioles and venules $[6,25,26,29,30]$, further studies, including conjunctival arterioles, are needed. Last, the sample size may be small. Thirty subjects were recruited in total; however, two of them missed the second visit due to conflicts in their schedules. Nevertheless, this study provided reasonable results which corroborated with previous studies of intra-visit variability [33].

\section{Conclusion}

In summary, the measurement variability of the vessel density of the bulbar conjunctiva appeared to have the smallest inter-visit variation. The measurement variability of the vasculature and circulation in HCL wearers were similar to that in NCL wearers. These findings are helpful in sample size calculation and study designs of further studies on conjunctival vascular responses to contact lens materials and designs.

\section{Acknowledgments}

All authors have no proprietary interest in any materials or methods.

\section{Funding}

Grant/financial support: This work was supported by a research grant provided by Johnson \& Johnson Vision Care, Inc.

Availability of data and materials

The datasets used and analyzed for the present study are available from the corresponding author.

\section{Authors' contributions}

JW, LH, CS and HJ collected and analyzed the data. JW, LH, CS and HJ interpreted the data. JW and $\mathrm{HJ}$ were the major contributors in writing the manuscript. All authors read and approved the final manuscript.

\section{Ethics approval and consent to participate}

All research methods are in accordance with the tenets of the Declaration of Helsinki and approved by the Human Subject Research Office (HSRO) at the University of Miami (ID: 20150359). All subjects that were recruited voluntarily were informed about the purposes, methods, and the potential risks of the study. A signed consent form was obtained from each volunteer.

Consent for publication

All study subjects gave informant consent.

Competing interests

The authors declare that they have no competing interests. 


\section{Author details}

'Miller School of Medicine, Bascom Palmer Eye Institute, University of Miami, 1638 NW 10th Avenue, McKnight Building - Room 202A, Miami, FL 33136, USA. ${ }^{2}$ School of Ophthalmology and Optometry, Wenzhou Medical University, Wenzhou, Zhejiang, China.

Received: 2 November 2018 Accepted: 11 March 2019

Published online: 01 April 2019

\section{References}

1. Guillon M, Shah D. Objective measurement of contact lens-induced conjunctival redness. Optom Vis Sci. 1996;73(9):595-605.

2. Murphy PJ, Lau JS, Sim MM, Woods RL. How red is a white eye? Clinical grading of normal conjunctival hyperaemia. Eye (Lond). 2007;21(5):633-8.

3. Peterson RC, Wolffsohn JS. Sensitivity and reliability of objective image analysis compared to subjective grading of bulbar hyperaemia. Br J Ophthalmol. 2007;91(11):1464-6.

4. MacKenzie LE, Choudhary TR, McNaught Al, Harvey AR. In vivo oximetry of human bulbar conjunctival and episcleral microvasculature using snapshot multispectral imaging. Exp Eye Res. 2016;149:48-58.

5. Chen W, Xu Z, Jiang H, Zhou J, Wang L, Wang J. Altered bulbar conjunctival microcirculation in response to contact lens wear. Eye Contact Lens. 2017;43(2):95-9.

6. Hu L, Shi $C$, Jiang H, Shi Y, Sethi Z, Wang J. Factors affecting microvascular responses in the bulbar conjunctiva in habitual contact lens wearers. Invest Ophthalmol Vis Sci. 2018:59(10):4108-14.

7. Ozkan J, Mandathara P, Krishna P, Sankaridurg P, Naduvilath T, Willcox MD, et al. Risk factors for corneal inflammatory and mechanical events with extended wear silicone hydrogel contact lenses. Optom Vis Sci. 2010;87(11):847-53.

8. Stapleton F, Carnt N. Contact lens-related microbial keratitis: how have epidemiology and genetics helped us with pathogenesis and prophylaxis. Eye (Lond). 2012;26(2):185-93.

9. Lim CH, Carnt NA, Farook M, Lam J, Tan DT, Mehta JS, et al. Risk factors for contact lens-related microbial keratitis in Singapore. Eye (Lond). 2016;30(3):447-55.

10. Efron N, Morgan PB. Rethinking contact lens associated keratitis. Clin Exp Optom. 2006;89(5):280-98.

11. Best N, Drury L, Wolffsohn JS. Predicting success with silicone-hydrogel contact lenses in new wearers. Cont Lens Anterior Eye. 2013;36(5):232-7.

12. McNally JJ, Chalmers RL, McKenney CD, Robirds S. Risk factors for corneal infiltrative events with 30-night continuous wear of silicone hydrogel lenses. Eye Contact Lens. 2003;29(1 Suppl):S153-6.

13. Stapleton F, Keay L, Edwards K, Naduvilath T, Dart JK, Brian G, et al. The incidence of contact lens-related microbial keratitis in Australia. Ophthalmology. 2008;115(10):1655-62.

14. Stapleton F, Keay L, Jalbert I, Cole N. The epidemiology of contact lens related infiltrates. Optom Vis Sci. 2007:84(4):257-72.

15. Efron N, Jones L, Bron AJ, Knop E, Arita R, Barabino S, et al. The TFOS international workshop on contact lens discomfort: report of the contact lens interactions with the ocular surface and adnexa subcommittee. Invest Ophthalmol Vis Sci. 2013;54(11):TFOS98-122.

16. Caffery B, Dogru M, Jones LW, Lin MC, Nichols JJ, Papas E, et al. Contact lens comfort. Optom Vis Sci. 2016;93(8):790-2.

17. Cheung AT, Hu BS, Wong SA, Chow J, Chan MS, To WJ, et al. Microvascular abnormalities in the bulbar conjunctiva of contact lens users. Clin Hemorheol Microcirc. 2012;51(1):77-86.

18. Dumbleton KA, Chalmers RL, Richter DB, Fonn D. Vascular response to extended wear of hydrogel lenses with high and low oxygen permeability. Optom Vis Sci. 2001;78(3):147-51.

19. Shi $Y, H u L$, Chen W, Qu D, Jiang H, Wang J. Evaluated conjunctival blood flow velocity in daily contact lens wearers. Eye Contact Lens. 2018;44(Suppl 1):S238-43.

20. Sorbara L, Maram J, Simpson T, Hutchings N. Corneal, conjunctival effects and blood flow changes related to silicone hydrogel lens wear and their correlations with end of day comfort. Cont Lens Anterior Eye. 2018;41(2):193-200.

21. Sorbara L, Simpson T, Duench S, Schulze M, Fonn D. Comparison of an objective method of measuring bulbar redness to the use of traditional grading scales. Cont Lens Anterior Eye. 2007;30(1):53-9.
22. McMonnies CW, Chapman-Davies A. Assessment of conjunctival hyperemia in contact lens wearers. Part I. Am J Optom Physiol Opt. 1987:64(4):246-50.

23. Schulze MM, Hutchings $N$, Simpson TL. The use of fractal analysis and photometry to estimate the accuracy of bulbar redness grading scales. Invest Ophthalmol Vis Sci. 2008;49(4):1398-406.

24. Khansari MM, Wanek J, Tan M, Joslin CE, Kresovich JK, Camardo N, et al. Assessment of conjunctival microvascular hemodynamics in stages of diabetic microvasculopathy. Sci Rep. 2017;7:45916.

25. Koutsiaris AG, Tachmitzi SV, Papavasileiou P, Batis N, Kotoula MG, Giannoukas $A D$, et al. Blood velocity pulse quantification in the human conjunctival pre-capillary arterioles. Microvasc Res. 2010;80(2):202-8.

26. Shahidi M, Wanek J, Gaynes B, Wu T. Quantitative assessment of conjunctival microvascular circulation of the human eye. Microvasc Res. 2010;79(2):109-13.

27. Jiang $\mathrm{H}$, Zhong J, DeBuc DC, Tao A, Xu Z, Lam BL, et al. Functional slit lamp biomicroscopy for imaging bulbar conjunctival microvasculature in contact lens wearers. Microvasc Res. 2014;92:62-71.

28. Deng Z, Wang J, Jiang H, Fadli Z, Liu C, Tan J, et al. Lid wiper microvascular responses as an indicator of contact lens discomfort. Am J Ophthalmol. 2016;170:197-205.

29. Koutsiaris AG, Tachmitzi SV, Batis N. Wall shear stress quantification in the human conjunctival pre-capillary arterioles in vivo. Microvasc Res. 2013:85:34-9.

30. Koutsiaris AG, Tachmitzi SV, Batis N, Kotoula MG, Karabatsas CH, Tsironi E, et al. Volume flow and wall shear stress quantification in the human conjunctival capillaries and post-capillary venules in vivo. Biorheology. 2007:44:375-86

31. Kwa VI, Lopez OL. Fractal analysis of retinal vessels: peeping at the tree of life? Neurology. 2010;74(14):1088-9.

32. Jiang H, DeBuc DC, Rundek T, Lam BL, Wright CB, Shen M, et al. Automated segmentation and fractal analysis of high-resolution non-invasive capillary perfusion maps of the human retina. Microvasc Res. 2013;89:172-5.

33. Xu Z, Jiang H, Tao A, Wu S, Yan W, Yuan J, et al. Measurement variability of the bulbar conjunctival microvasculature in healthy subjects using functional slit lamp biomicroscopy (FSLB). Microvasc Res. 2015;101:15-9.

34. Burgansky-Eliash Z, Lowenstein A, Neuderfer M, Kesler A, Barash H, Nelson DA, et al. The correlation between retinal blood flow velocity measured by the retinal function imager and various physiological parameters. Ophthalmic Surg Lasers Imaging Retina. 2013;44(1):51-8.

35. Koutsiaris AG. Hemodynamics in the microcirculation. Ann Biomed Eng. 2016:44(4):1321-2.

Ready to submit your research? Choose BMC and benefit from

- fast, convenient online submission

- thorough peer review by experienced researchers in your field

- rapid publication on acceptance

- support for research data, including large and complex data types

- gold Open Access which fosters wider collaboration and increased citations

- maximum visibility for your research: over $100 \mathrm{M}$ website views per year

At $\mathrm{BMC}$, research is always in progress.

Learn more biomedcentral.com/submissions 